\section{REALIDAD AUMENTADA, EDUCACIÓN Y MUSEOS}

\section{David Ruiz Torres}

\section{Licenciado en Historia del Arte e Historia}

Facultad de Filosofía y Letras. Universidad de Granada. Campus Universitario de Cartuja, s/n. 18071 Granada (España) - Email: druiztorres@ugr.es

\section{Resumen}

En los últimos años la Realidad Amentada está consiguiendo un protagonismo cada vez más importante en diversas áreas de conocimiento, mostrando la versatilidad y posibilidades que presenta esta nueva tecnología derivada de la Realidad Virtual.

La capacidad de insertar objetos virtuales en el espacio real y el desarrollo de interfaces de gran sencillez, la han convertido en una herramienta muy útil para presentar determinados contenidos bajo las premisas de entretenimiento y educación, en lo que se conoce como "edutainment".

Fruto de esa dimensión educativa que representa, se han desarrollado en nuestro país varios proyectos para las aulas como pueden ser “Aprendra" (Valencia), o el denominado "Big-Bang 2.0" dentro del programa Eskola 2.0 (País Vasco).

La Realidad Aumentada también ha demostrado su función pedagógica en otro tipo de escenarios como son los museos y centros de interpretación, donde constituye uno de los recursos museográficos más vanguardistas gracias a que favorece la interacción entre los visitantes y el objeto cultural de una forma atractiva a la vez que didáctica.
Palabras clave

Realidad Aumentada, educación, museos, difusión, interacción, edutainment

Key Words

Augmented Reality, education, museums, diffusion, interaction, edutainment

Abstract
Nowadays, Augmented Reality is
getting a more predominant role in
different areas of knowledge, showing
the versatility and possibility this new
technology derived from virtual
reality presents.

The capacity to insert virtual objects in real space and the development of user interfaces of great simplicity have made possible that Augmented Reality

has become a useful tool to present certain content under the premises of entertainment and education, which is known as "edutainment".

Various Spanish projects have been developed for the classroom according to this educational dimension, such as

"Aprendra" (Valencia), or the socalled "Big Bang 2.0" in the program

Eskola 2.0 (Basque Country).

Augmented Reality has also shown its pedagogical function in other settings such as museums and interpretations centers.

The most avant-garde museography resources make use of this technology in favor of the interaction between the visitors and the cultural object in an attractive and educative experience.

This is the case of The Military Order of Calatrava Interpretation Center 
Es el caso del Centro de Interpretación de la Orden Militar de Calatrava (Alcaudete, Jaén) o el Centro de Interpretación de la Tecnología (Zamudio, Bizcaia), que cuentan en su discurso expositivo con instalaciones basadas en esta tecnología, cumpliendo con la principal función de estos espacios: la difusión de contenidos culturales.
(Alcaudete, Jaén), or the Technology Interpretation Center (Zamudio, Bizcaia), which have faculties based on this technology, achieving the main function of these spaces: the diffusion of cultural content.

\section{Introducción}

La Realidad Aumentada representa actualmente una potente herramienta que ha mostrado su versatilidad en un amplio abanico de aplicaciones en diferentes áreas de conocimiento. Una de ellas ha sido el campo educativo donde ha encontrado grandes posibilidades para la difusión y conocimiento de contenidos que se presenta de una forma atractiva y pedagógica al mismo tiempo.

Los museos y centros de interpretación se han erigido en la actualidad como otro espacio para la educación donde las nuevas tendencias museográficas han evolucionado desde la concepción de galería heredada del siglo XIX, a la de constituir centros para la transmisión del conocimiento en la que los visitantes no actúan como meros observadores pasivos sino que interactúan con los contenidos que se presentan bajo un enfoque lúdico-racional, y que especialmente están siendo explotados para los colectivos más jóvenes de nuestra sociedad.
Entre los recursos que se han utilizado para la consecución de esta nueva orientación de los espacios museísticos, las nuevas Tecnologías de la Información y Comunicación (TICs), se presentan como un importante filón por el grado de cotidianidad que presentan para la sociedad actual, ofreciendo importantes resultados en combinación con las técnicas museográficas tradicionales.

La Realidad Aumentada ha aparecido con gran fuerza dentro de este contexto en los últimos años, ya que ofrece grandes posibilidades por su atractivo y capacidad de insertar objetos virtuales en un espacio real. Como muestra del establecimiento de esa relación ha sido el crecimiento de publicaciones que abordan esta temática, así como los casos concretos de aplicabilidad en los que se han incluido instalaciones que utilizan esta nueva tecnología dentro de los programas museográficos. 


\section{Objetivos}

Con carácter general, se pretende plantear en este artículo la aparición de un nuevo recurso dentro de las Tecnologías de la Información y Comunicación (TICs), como es la Realidad Aumentada, y su aplicación dentro de contextos educativos en el ámbito español, prestando especial atención a los entornos expositivos y museísticos.

Para ellos se habrá de estudiar los diferentes modos de interacción que se desarrollan a través de las interfaces persona - ordenador, que se utilizan para las aplicaciones de Realidad Aumentada.
También, realizar un juicio sobre cuáles serán los más recurrentes dentro de las aplicaciones de Realidad Aumentada en entornos educativos y demarcar sus posibilidades, así como sus ventajas e inconvenientes.

Finalmente se exponen aquellos casos concretos en los que se han realizado experiencias con Realidad Aumentada dentro del Patrimonio Cultural español, realizando una labor de prospección que nos muestre el grado de aplicabilidad que se ha efectuado desde su aparición.

\section{Metodología}

El ámbito de actuación ha abarcado las experiencias realizadas en nuestro país desde la aparición de la Realidad Aumentada, con el fin de establecer una evaluación hasta la fecha en el que se pondrá especial interés en las investigaciones y proyectos realizados al respecto, y por otra parte, los ejemplos concretos que se encuentran ya desarrollados y aplicados dentro de entornos educativos en España. Una vez entendida la tecnología de Realidad Aumentada en su concepto y funcionamiento, el estudio de las aplicaciones de dicha tecnología dentro de los diferentes proyectos educativos ha sido la línea a seguir como primer punto antes de centrarnos en los casos de entornos museísticos. Por otra parte se realizaron otra serie de visitas a los diferentes museos y centros de interpretación en los que existen instalaciones de Realidad Aumentada dentro del discurso museográfico de los mismos. Igualmente se ha prestado atención a las empresas que trabajan con Realidad Aumentada, que son las encargadas de llevar a la práctica aquellas experiencias y proyectos que surgen dentro de ámbitos académi$\cos$ e investigadores y que asesoran a las mismas para su incursión en el mercado y en definitiva, hacen llegar los nuevos avances tecnológicos al gran público. Se ha evaluado especialmente aquellas que tienen una relación con ámbitos educativos, bien a través de los proyectos museográficos, propuestas para el aula, o de difusión que han encontrado en la Realidad Aumentada 


\section{La Realidad Aumentada como herramienta en contextos educativos}

\subsection{Hacia una definición de Realidad Aumentada}

La Realidad Aumentada, en su desarrollo, ha seguido un itinerario ligado a otro concepto mucho más conocido en nuestra sociedad como es la Realidad Virtual, aunque los progresos y avances realizados durante los últimos años han hecho que surja como una nueva tecnología con entidad propia. Desde que en 1992 se acuñara el término Realidad Aumentada (Caudell, 1992), han sido numerosas las investigaciones al respecto que, junto a los avances científicos y tecnológicos, han dado lugar a su maduración y aplicación en diferentes áreas apareciendo diferentes monografías que versan sobre la misma (Bimber, 2005; Haller, 2007; Hainich, 2010). Paul Milgram y Fumio Kishino definieron en su denominado "continuo de la virtualidad" (1994), lo que se conoce como Realidad Mezclada, en la que los elementos virtuales se combinan con el espacio físico real.
Gráfico $n^{\circ}$ 1: Continuo de la virtualidad

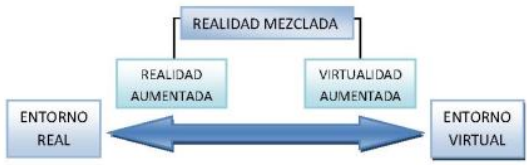

Fuente: Milgram, P., Kishino, F. (1994)

Posteriormente el trabajo de Ronald Azuma (1997), codificaría las diferentes características que permiten su definición $\mathrm{y}$ diferenciación de la Realidad Virtual. Azuma consideró la Realidad Aumentada como aquella que permite al usuario ver en todo momento el mundo real, al que se le superponen objetos virtuales coexistiendo ambos en el mismo espacio. En una publicación posterior (2001), determinó más concisamente sus características basándose en tres pilares básicos: combinar lo real y lo virtual en un entorno real, interactividad en tiempo real, y registro en 3D.

De modo que, esta tecnología se caracteriza por combinar el mundo real con el virtual, de manera que en este caso lo que ocurre es que a nuestra visión del mundo real se añaden contenidos virtuales generados por ordenador. Esto es posible gracias a la utilización de un display que puede ser desde unas gafas de Realidad Aumentada o 
Head Mounted Displays (HMDs), a dispositivos móviles tales como PDAs, PCs Ultramóviles, o teléfonos móviles, los cuales están teniendo actualmente un alto grado de aplicabilidad de la Realidad Aumentada, al igual que numerosas opciones que han hecho más cercana esta nueva tecnología. Así, a través de la cámara de cualquiera de estos dispositivos es posible obtener una imagen aumentada o enriquecida de aquello que observamos mediante la superposición de información virtual ${ }^{\mathrm{i}}$.

\subsection{Realidad Aumentada en el aula}

Esta capacidad de mezclar el mundo real con el virtual ofrece grandes posibilidades en el campo de la educación, como lo demuestran las experiencias realizadas hasta la fecha.

Una de las experiencias más recurrentes han sido aquellas basadas en la metáfora del libro aumentado, empleada sobre todo en aplicaciones relacionadas con entornos educativos. Así, a partir de un marcador impreso en una de las páginas, es posible acceder a información adicional mediante gráficos 3D, que muestran figuras virtuales que aparecen sobre las páginas del libro y que se visionan a través de la pantalla de un simple ordenador con webcam. Uno de los casos más significativos, al constituir una de los primeros ensayos, fue desarrollado por el Human Interface Technology Laboratory de la Universidad de Washington, que presentó el denominado Magic Book, que mostraba el valor didáctico de esta tecnología y el gran atractivo que supone en contextos educacionales (Billinghurst et al., 2001).

\section{Gráfico n ${ }^{\circ}$ 2: Aplicación de Realidad} Aumentada "Magic Book"

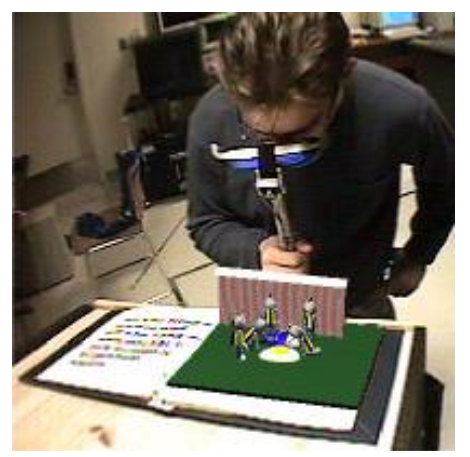

Fuente: Billinghurst, M., Kato, H., Poupyrev, I. (2001)

Esta misma aplicación ha sido desarrollada en nuestro país con el denominado "Libro Interactivo de Monumentos Andaluces", creado por la empresa malagueña ArpaSolutions, que contenía en sus páginas interiores diferentes marcadores que al visionarlos a través de una webcam, era posible observar las reproducciones virtuales tridimensionales en la pantalla del ordenador, ofreciendo pequeñas maquetas que los usuarios podían manipular como si se tratara de una real, acercando o moviendo el libro respecto a la cámara (Ruiz et al., 2007).

También se han realizado varias experiencias similares que muestran la incursión de esta tecnología y su fiabilidad. Es el caso del proyecto "Big-Bang 2.0", desarrollado 
por la empresa VirtualWare, y que se encuadra dentro del programa Eskola 2.0, perteneciente al Departamento de Educación, Universidades e Investigación del Gobierno Vasco (Wirtualware, 2010). Éste pretende fomentar la utilización de materiales didácticos digitales para la materia de Conocimiento del Medio del tercer ciclo de Educación Primaria, para ser utilizados en el aula con el uso de los nuevos recursos docentes de reciente implantación como son las pizarras digitales $\mathrm{u}$ ordenadores portátiles. La Realidad Aumentada forma parte de los recursos que se utilizan para el proyecto en el que se pueden obtener objetos virtuales como si fueran reales.

Igualmente encontramos el proyecto "APRENDRA", desarrollado por El grupo de Gráficos y Multimedia del Instituto de Automática e Informática Industrial (ai2) y l'Escola d'Estiu de la Universidad Politécnica de Valencia, junto con el Instituto Tecnológico del Juguete (AIJU) de Ibi (Alicante). Aquí se pretende utilizar la tecnología de Realidad Aumentada para elaborar juegos educativos para el aula mediante la fórmula educación + entretenimiento conocida como "edutainment". Los primeros resultados han consistido en implementar una aplicación para iphone en la que a través de unos marcadores, es posible observar a través de la pantalla diferentes modelos virtuales 3D que se corresponden con la flora, fauna y monumentos de Asia, África y América Central y del Sur (Aprendra, 2010). La aplicación ha sido testada con alumnos de tercero y cuarto de Educación Primaria, para los que se han desarrollado diferentes juegos educativos que utilizan la tecnología de Realidad Aumentada para favorecer la interacción entre el niño y los modelos virtuales.

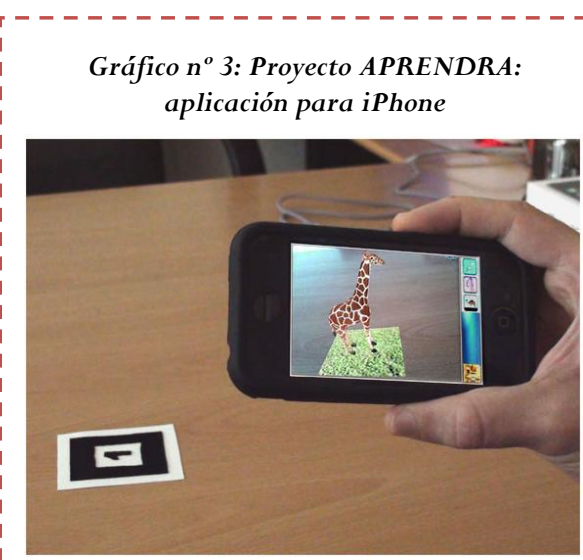

Fuente: http: / / www.aprendra.es / [consultado: 20.01.2011]

La iniciativa de APRENDRA resultar ser un recurso muy didáctico para mostrar a los escolares aquellos contenidos con los que se encuentran menos familiarizados, como son las especies y construcciones de países no-occidentales, permitiendo obtener una visión multicultural a través de una experiencia lúdica.

Otra aportación importante es la desarrollada en la Universidad Jaime I de Castellón, por los Departamentos de Química Inorgánica y Orgánica y el Departamento de Lenguajes y Sistemas Informáticos, que presentaron un recurso de Realidad $\mathrm{Au}$ mentada para las asignaturas del área de conocimiento de Química Inorgánica. Aquí 
se diseñaron varios modelos virtuales tridimensionales de ciertas estructuras cristalinas de difícil comprensión por lo abstracto de su composición, que a través de la Realidad Aumentada se pudieron manipular como si fueran objetos reales a través de diferentes marcadores colocados en una pieza irregular. La experiencia se desarrolló conjuntamente a las clases docentes permitiendo a los alumnos una mejor comprensión mediante la manipulación de las mismas (Núñez et al., 2008).

Otras experiencias de Realidad Aumentada en el área educativa han estado relacionadas con la didáctica musical. Así, como resultado de una colaboración entre el grupo ISIS (Departamento de Tecnología Electrónica) y el Departamento de Ingeniería de Comunicaciones de la Universi- dad de Málaga, se presentaron en la VI Semana de la Ciencia de Andalucía las aplicaciones "Piano Virtual" y "Partitura Virtual” (Peula et al., 2007).

En la primera se encontraba la plantilla de un teclado sobre papel que podía tocarse como si fuera un piano real a través de la imagen capturada por una webcam que a través de unos altavoces, reproducía los sonidos según las "teclas" que se tocaran en la plantilla. En el segundo caso, era posible crear una melodía colocando notas musicales sobre un pentagrama virtual. Aquí, las diferentes notas estaban representadas por marcadores que se correspondían con valores diferentes que al ser reconocidos por la cámara representaban su valor sobre la partitura virtual que se visionaba en la pantalla del PC.

\section{La Realidad Aumentada y su implantación en entornos museísticos}

\subsection{Museos y Centros de Interpretación: espacios para la experiencia aumentada}

Por otra parte, en España se han producido varios casos de aplicaciones de Realidad Aumentada en espacios museísticos, especialmente durante el año 2010, que representan un punto de partida en cuanto a la utilización de este nuevo recurso, en vista de los nuevos desarrollos que se producen simultáneamente a la publicación del presente artículo.

Uno de los ejemplos que podemos citar es el Centro de Interpretación de la Orden Militar de Calatrava que se encuentra en un escenario singular como es el Castillo de Alcaudete en Jaén. Aquí la visita al conjunto comienza con una instalación de Realidad Aumentada en la que se ofrece a través de unas maquetas virtuales el proceso constructivo de la fortaleza desde sus primeros asentamientos hasta su abandono 
como tal. Dentro de las inmediaciones de un aljibe se encuentran dos pantallas sobre atril con una cámara web incorporada, delante de las cuales se muestran unas bandejas circulares que contienen unos marcadores con patrones impresos que el sistema reconocerá a través de la imagen de vídeo.

Gráfico $n^{o}$ 4: Centro de Interpretación de la Orden Militar de Calatrava. Instalación de Realidad Aumentada que muestra la evolución constructiva de la fortaleza
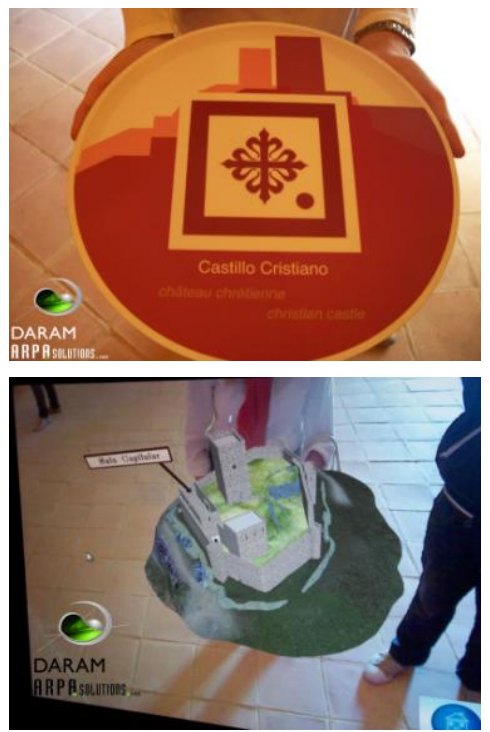

Fuente: Arpa-Solutions, S.L. Plataforma Software DARAM® de Realidad Aumentada (C)Arpa-Solutions.

En la pantalla se mostrará una maqueta virtual animada que mostrará el proceso constructivo del conjunto de forma cronológica, junto a etiquetas flotantes que indicarán las partes que lo componen. Así, desde la construcción del alcázar islámico primigenio se puede ver la evolución a través de la fortaleza calatrava y finalmente la transformación en residencia palacial renacentista de los Fernández de Córdoba.

El Museo de la Autonomía de Andalucía (Coria del Río, Sevilla), situado en el entorno de la Casa de Blas Infante, también cuenta dentro de la sala de exposición permanente con una instalación basada en Realidad Aumentada que muestra las diferentes instituciones andaluzas. Aquí aparecen unos modelos virtuales tridimensionales de los edificios donde se ubican como son el Parlamento Andaluz, el Consejo de Gobierno, ambos en Sevilla, y el Tribunal Superior de Justicia de Andalucía en Granada. Mediante una aplicación similar a través de unas bandejas circulares con unos marcadores o patrones impresos, se colocan delante de una cámara web mientras que en la pantalla aparecen las maquetas virtuales sobre los marcadores que portan los visitantes. En la base de cada edificio aparecen etiquetas con información sobre la institución andaluza que representa y sus funciones gubernamentales, además de la historia del edificio donde se ubican. La intención de esta aplicación es la de dar a conocer a los visitantes los órganos de gobierno que los representan así como la funcionalidad que contienen cada uno de ellos. 


\section{Gráfico no 5: Museo de la Autonomía} de Andalucía. Usuario interactuando con la aplicación sobre las instituciones representativas andaluzas


Fuente: Arpa-Solutions, S.L. Plataforma Software $D A R A M \circledR$ de Realidad Aumentada. (CArpa-Solutions.

Ambos casos representan un ejemplo de las posibilidades educativas que ofrece esta nueva tecnología, debido a su fácil manejo ya que no se encuentra ningún dispositivo hardware entre el usuario y la aplicación, sino que la interacción de produce de una forma directa en la que los visitantes son capaces de relacionarse con los contenidos de una forma aprehensiva manipulando los modelos virtuales como si se tratara de objetos reales. Las posibilidades de animación virtual o la inclusión de etiquetas flotantes ofrecen también un importante recurso para añadir información adicional que supera las tradicionales cartelas o pa- neles informativos. Por otra parte, muchos de los contenidos que se pretenden transmitir muestran una complejidad que una representación aumentada soluciona de una forma atractiva e instructiva al poder añadir los contenidos virtuales fácilmente.

Otros ejemplos de esa relación entre Realidad Aumentada y educación en centros museísticos ha sido llevado a cabo en el caso del Centro de la Interpretación de la Tecnología, situado dentro del Parque Tecnológico de Bizkaia (Zamudio, Bizkaia), surgido con la intención de acercar a los visitantes, principalmente estudiantes de Educación Secundaria, aquellos avances tecnológicos que se desarrollan en los centros de investigación que se hallan en el entorno. Aquí se encuentran un gran número de aplicaciones para interactuar con los visitantes y enseñar en qué consisten esos avances relacionados con la domótica, la nanotecnología, la biotecnología, o la robótica. Dentro de los recursos museográficos se encuentra una instalación de Realidad Aumentada que enseña el funcionamiento de las denominadas "cajas negras", mediante unas bandejas con marcadores que representan modelos virtuales de varios aparatos eléctricos junto a información adicional animada que ayuda a comprender el funcionamiento del mismo $\mathrm{y}$ para qué sirve. La labor de este Centro de Interpretación pretende ser un complemento a las clases dentro del aula y además fomentar dentro de los visitantes más jóvenes el espíritu crítico y el conocimiento de 
aquellas líneas de investigación más punteras que se están desarrollando actualmente.

\subsection{Museos Virtuales y Realidad Aumentada: el museo en tus manos}

Otra aportación importante ha tenido lugar en el Museo Virtual de la Informática, emplazado en el edificio Fermín Caballero de la Escuela Superior de Informática de la Universidad de Castilla - La Mancha (Ciudad Real), que ha desarrollado un proyecto de museo virtual en un punto de información dentro del edificio (Grupo Oreto/Arco, 2009). En éste es posible acceder a contenidos multimedia e información sobre el recorrido histórico de esta joven ciencia, y también interactuar con modelos virtuales tridimensionales de equipos que ya han entrado a formar parte de la historia, a través de una aplicación de Realidad Aumentada basada en marcadores. Este recurso ofrece grandes posibilidades tanto en su implantación en el museo in situ como en las posibilidades que ofrece en su versión online. Así, mediante la aplicación es posible aumentar la colección de las piezas que se pueden observar en el Museo de la Informática, al igual que permite interactuar con las mismas completando la mera observación pasiva de los visitantes en las tradicionales vitrinas.

Relacionado con la idea de este proyecto es necesario destacar que el caso de los museos virtuales ofrece grandes posibilidades para las aplicaciones de Realidad Aumen- tada dentro del entorno doméstico. La sencillez de las interfaces persona-ordenador que se establecen a través de esta tecnología, permiten que a través de dispositivos con los que estamos familiarizados habitualmente sean utilizados para obtener una experiencia didáctica online. Uno de los ejemplos que se han llevado a cabo en nuestro país, ha sido el Museo del Jurásico de Asturias que, en colaboración con el diario Público, ofrece la posibilidad de interactuar con varios especímenes de dinosaurios virtuales. Entrando en la web http://www.publico.es/especial/dinosaurios/, e imprimiendo uno de los patrones que se pueden descargar desde la misma, se colocan sobre una superficie y al ser enfocados por la cámara web aparecerá un dinosaurio virtual que además puede caminar y moverse utilizando los controles del teclado.

Esta experiencia, conseguiría un propósito fundamental en esta tipología de museos que sería de la difusión, generando visitantes potenciales que se interesen mediante el uso de la aplicación.

\section{Gráfico $n^{\circ}$ 6: Aplicación on-line pertene-} ciente al Museo del Jurásico de Asturias.

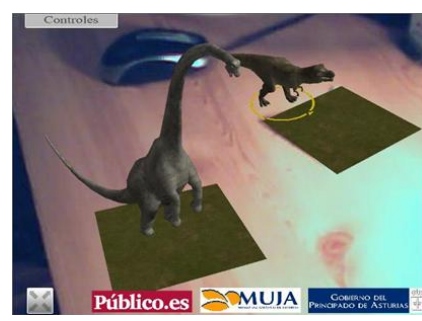

Fuente: Imagen capturada por el autor 


\subsection{Realidad Aumentada: una apuesta de futuro}

Así, la Realidad Aumentada, ofrece la posibilidad de ofrecer nuevos contenidos a través de la red, ya sea de un museo o una exposición, ofreciendo interactuar con algunas de las piezas o los contenidos virtualmente y como si se tratara de objetos reales manipulando el marcador correspondiente. El carácter didáctico de este tipo de aplicaciones queda manifiesto y supone una llamada a visitar el lugar del que proceden o, en otro caso, disfrutar de aquellos contenidos que se encuentran en museos a miles de kilómetros, o que ya han desaparecido en el caso de las exposiciones temporales.

Dentro del proyecto SIAMA también se presentaron algunas propuestas relacionadas con aplicaciones de Realidad Aumentada en museos, con motivo de la VII Semana de la Ciencia y la Tecnología de Andalucía, que tuvo lugar en el año 2007 en Málaga (Peula et al., 2007b). Una de ellas consiste en la recreación del interior de una sala de un templo egipcio en la que se encontraba un relieve procedente de la capilla de la diosa Hator, en el templo de Hatseshut en Deir el-Bahari. El visitante entraba a la sala totalmente a oscuras, portando una "linterna mágica" con la que era capaz de descubrir cada parte del relieve sobre el que aparecía información textual sobre la escena "iluminada" como procedencia, cronología, iconografía, etc. La interfaz consistía en la "linterna mágica" que con- tenía un marcador, que al ser reconocido por la cámara situada sobre la pantalla sobre la que se proyectaba el relieve egipcio, hacía que se iluminase la parte del mismo al que se orientaba la linterna.

La misma solución se aplicó a una pintura renacentista que representa a "La Virgen, el Niño y San Juan" atribuida al taller de Pietro Perugino y que se encuentra en la $\mathrm{Na}$ tional Gallery de Londres. Aquí se recurrió a una radiografía realizada en una restauración previa de la obra en la que podían observarse ciertos detalles sobre su elaboración. La "linterna mágica" se proyecta sobre la pintura "descubriendo" una visión de rayos $\mathrm{X}$, en la que se explicaban detalles como arrepentimientos, soportes de sujeción, técnicas empleadas, etc.

\section{Gráfico $n^{\circ}$ 7: Recreación ideal de la} instalación museística del relieve egipcio del templo de Hatseshut

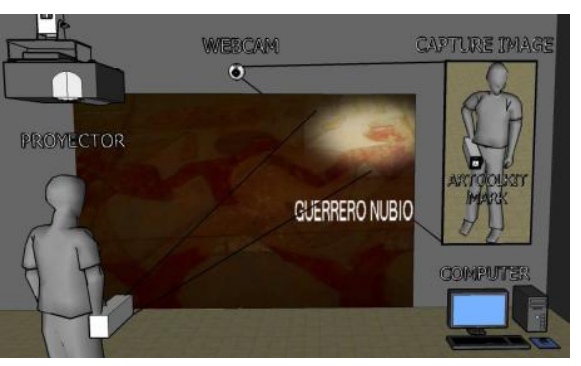

Fuente: Peula, J. M., Torres, F., Urdiales, C., Sandoval, F. (2008) 
En estos ensayos se mostró la versatilidad que la Realidad Aumentada puede presentar en su aplicación en espacios museísticos, ofreciendo recursos de un alto grado pedagógico que ayudan a que la obra "hable" al visitante, y en definitiva ofrezca un mayor contenido. El atractivo de la aplicación, permite a los usuarios interactuar con la obra de una forma sencilla en la que prima el valor didáctico.

\section{Conclusiones}

A pesar de que la Realidad Aumentada es una gran desconocida para el público en general, los últimos años han sido decisivos para adentrarse en el actual contexto social, dentro del cual los entornos educativos están cobrando un gran protagonismo. Aquí la Realidad Aumentada se utiliza como una herramienta de comunicación y difusión de los contenidos, en los que prima un enfoque lúdico y racional que resulta de gran atractivo. En esta afirmación, y en las experiencias comentadas en el presente trabajo, la utilización de la tecnología no debe eclipsar esos contenidos que son la fuente fundamental de su aplicación en entornos educativos, sin dejar que la tecnología invada u oculte lo que se quiere definir.

Las aplicaciones que se insertan en contextos educativos, son aquellas que están basadas en las denominadas interfaces tangibles de usuario que permiten manipular un objeto real a través de marcadores como si se tratase de un objeto real. El hecho de que sean las elegidas para este tipo de entornos, es que la interacción entre el usuario y el objeto virtual se produce de una forma natural e intuitiva, sin ningún tipo de hardware adicional, que medie en la relación persona-ordenador.

Referente a su proyección dentro de este tipo de entornos, la utilización de un sistema basado en componentes que se encuentra fácilmente en el mercado (ordenador, cámara, pantalla, etc.), ofrece otro tipo de ventajas que hacen de éstas un recurso económico y de fácil mantenimiento que se añade a su valor didáctico.

Por otra parte, se han observado las posibilidades que la Realidad Aumentada ofrece en entornos museísticos atendiendo a su carácter pedagógico, ya que la sencillez de las aplicaciones hace que se adapten a un amplio espectro de público que no tiene que estar familiarizado con las nuevas tecnologías

En los casos estudiados se ha podido observar que los planteamientos museográficos más actuales incluyen la Realidad Aumentada como una herramienta capaz de mostrar al visitante unos contenidos de una forma atractiva y didáctica al mismo tiempo, haciendo que el visitante se convierta en protagonista de la instalación y no sola- 
mente actúa como observador pasivo dentro del entorno museístico.

Bajo esta premisa, el uso de la Realidad Aumentada se materializa con el fin de ofrecer una visión más completa de lo que se exhibe o nuevas interpretaciones que ayuden a comprender mejor los objetos expuestos, que favorecen la experiencia que se puede obtener en este tipo de espacios.

Como hemos mencionado, la mayoría de aplicaciones de Realidad Aumentada citadas, cumplen una labor fundamentalmente didáctica, resaltando especialmente aquellas que se encuentran en entornos expositivos. Tras nuestro estudio, se puede afirmar que estas experiencias están enfocadas a un público joven mayoritariamente, ya que se encuentran familiarizados con las nuevas tecnologías y el uso de los dispositivos empleados. La Realidad Aumentada presenta una configuración novedosa $y$ atractiva que permite transmitir unos contenidos bajo la fórmula educación + diversión que se pretende en este tipo de entornos.

Finalmente, haremos referencia de forma general al concepto que se transmite en el presente artículo acerca de la Realidad Aumentada y las posibilidades que ofrece: acceder al conocimiento con una nueva óptica, en la que prima la variedad del discurso interpretativo, y puesta en valor del mismo. La labor más importante desde nuestra disciplina referente a la Realidad Aumentada, será la de transmitir la necesidad que presentan desde nuestra perspectiva esos entornos educativos y museísticos, con el objetivo de que los instrumentos aportados por la ciencia nos permitan acceder a un conocimiento más amplio y diversificado.

\section{Referencias}

APRENDRA (2010). "La UPV y AIJU presentan el proyecto 'APRENDRA' para aprender jugando con la Realidad Aumentada”. Nota de prensa. En: http://www.aprendra.es/antigua/archivos/nota p rensa aprendra.pdf [consultado: 17.11.2010].

AZUMA, R. (1997), "A Survey of Augmented Reality". En: Presence: Teleoperators and Virtual Environments, vol. 6, no 4, pp. $355-385$,

http: / / citeseerx.ist.psu.edu/viewdoc/download?do

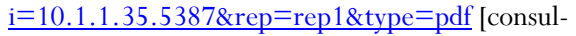
tado: 16.11.2010].

AZUMA, R., BAILLOT, Y., BEHRINGER, R., FEINER, S., JULIER, S., MACINTYRE, B. (2001).

"Recent Advances in Augmented Reality". En: IEEE Comput. Graph. Appl. 21, 6, pp. 34-47, http://ieeexplore.ieee.org/stamp/stamp.jsp?tp=\& arnumber $=963459$ \&userType $=$ inst [consultado: 15.11.2010].

BILLINGHURST, M., KATO, H., POUPYREV, I. (2001). "The MagicBook: A transitional AR interface". En: Elsevier Computers and Graphics, vol. 25, pp. 745 - 753, http://www.hitl.washington.edu /people/tfurness/courses/inde543/READINGS03/BILLINGHURST/MagicBook.pdf [consultado: 26.12.2010].

BIMBER, O., RASKAR, R. (2005). Spatial augmented reality: merging real and virtual worlds. Wellesley, Mass.: A K Peters.

CAUDELL, T. P., MIZELL, D.W. (1992). "Augmented Reality: An Application of Heads-Up Dis- 
play Technology to Manual Manufacturing Processes”. En: International Conference on System Sciences, Kauai, Hawaii, vol. 2, pp. 659-669,

http:/ /ieeexplore.ieee.org/stamp/stamp.jsp?arnum ber $=00183317$ [consultado: 25.12.2010].

GRUPO ORETO/ARCO (2009). "Realidad Aumentada. Proyecto Museo Virtual”. En: Síntesis de Imagen Digital 3D. Aplicaciones y Portfolio de Proyectos. Escuela Superior de Informática, Universidad de Castilla - La Mancha, p. 5, http://www.infcr.uclm.es/www/cglez/downloads/projects/3D ArcoOreto.pdf [consultado: 03.01.2011].

HAINICH, R. (2010). The End of hardware: augmented reality and beyond. [S. 1.]: Springer.

HALLER, M., BILLINGHURST, M., THOMAS B. H. (2007). Emerging technologies of augmented reality: interfaces and design. Hershey : Idea Group Pub.

MILGRAM, P., KISHINO, F. (1994). "A Taxonomy of Mixed Reality Visual Display". En: Inst. of Electronics, Information and Communication Engineers (IEICE) Trans. Information and Systems, vol. E77-D, $\mathrm{n}^{\circ}$. 12, pp. 1321-1329, http: / / citeseerx.ist. psu.edu/ viewdoc/download?doi=10.1.1.102.

$\underline{4646 \& \text { rep }=\text { rep } 1 \& \text { type }=\text { pdf }}[$ consultado: 15.11.2010].

NÚÑEZ, C., NÚÑ̃Z, M., CARDA, J. B., QUIRÓS, R. J. (2008). "Interactuando con las estructuras cristalinas. Realidad Aumentada aplicada al estudio y comprensión de estructuras cristalinas tridimensionales en Química Inorgánica”. En: III Reunión INDOQUIM 2008, Innovación Docente en Química. Servicio de Publicaciones de la UCA
(Universidad de Cádiz), pp. 149 - 150, http: / / www.indoquim.org/actas/Indoquim 2008 Actas.pdf [consultado: 18.01.2011].

PEULA, J. M., TORRES, F., URDIALES, C., SANDOVAL, F. (2008). "Aplicación de Realidad Aumentada para la educación y difusión del patrimonio". Artículo presentado en el XXII Simposium Nacional de la Unión Científica Internacional de Radio (URSI 2008) celebrado en Madrid. En:

http: / / www.siama.uma.es/index.php?option $={ }_{\text {com }}$ docman\&task $=$ doc download \& gid $=41 \&$ Itemid $=4$ o [consultado: 25.01.2011].

PEULA, J. M., ZUMAQUERO, J. A., URDIALES, C., BARBANCHO, A. M., SANDOVAL, F. (2007). "Realidad Aumentada aplicada a herramientas didácticas musicales”. En: http: / / www .grupoisis.uma.es/administrator/comp onents/com_jresearch/files/publications/ursi2007 26.pdf [consultado: 13.01.2011].

RUIZ, A., ACIÉN, F., VÁZQUEZ, J.L. (2007). "Sistemas de posicionamiento en la creación de un libro interactivo". En: Revista Digital Universitaria, Universidad Nacional Autónoma de México, vol. 8, no 10, pp. 1-9, http: / / www.revista.unam.mx /vol.8/num6/art49/jun art49.pdf [consultado: 14.01.2011].

VIRTUALWARE (2010). "Comienza el despliegue tecnológico del programa Eskola 2.0”. En:

http://www. virtualware.es/es/noticia/actualidadvirtualware/comienza-el-despliegue-tecnologicodel-programa-eskola-2-0.aspx [consultado: 10.11.2010].

\section{Cita de este artículo}

RUIZ TORRES, D. (2011) Realidad Aumentada, educación y museos. Revista Icono14 [en línea] 1 de julio de 2011, Año 9, Volumen 2. pp. 212-226. Recuperado (Fecha de acceso), de http://www.icono14.net 


\section{NOTAS}

${ }^{i}$ Hemos de señalar que en muchas de las definiciones de Realidad Aumentada que encontramos en la bibliografía, únicamente se refieren a sistemas visuales, que aunque se trata de una tecnología eminentemente visual y donde tiene un gran desarrollo, actualmente existe un gran interés en la investigaciones sobre la materia en la posibilidad de crear sistemas de Realidad Aumentada de tipo sonoro, háptico o multimodal.

${ }^{i i}$ El proyecto APRENDRA está financiado por el Ministerio de Ciencia e Innovación, bajo el título de Desarrollo y Validación de Sistemas de Realidad Aumentada para el Aprendizaje-Entretenimiento, teniendo prevista su finalización en diciembre de 2012. Web de APRENDRA: www.aprendra.es 